\title{
Effects of melanoma differentiation associated gene-7 (MDA-7/IL-24) on apoptosis of liver cancer cells via regulating the expression of $B$-cell lymphoma-2
}

\author{
QINGGUO MO ${ }^{1}$, LIN LIU ${ }^{1}$, GUANGHE BAO ${ }^{2}$ and TONGFEI LI ${ }^{3}$ \\ ${ }^{1}$ Department of Interventional Therapy, The Third Affiliated Hospital of Qiqihar Medical University, \\ Qiqihar, Heilongjiang 161000; ${ }^{2}$ Department of Interventional Therapy, Qinghai Provincial People's Hospital, \\ Xining, Qinghai 810000; ${ }^{3}$ Department of Interventional Therapy, Affiliated Hospital of Taishan Medical University, \\ Taian, Shandong 271000, P.R. China
}

Received November 19, 2018; Accepted March 26, 2019

DOI: $10.3892 / \mathrm{ol} .2019 .10298$

\begin{abstract}
The present study investigated the mechanism of selective killing of liver cancer cells of melanoma differentiation associated gene-7 (MDA-7, also called IL-24 $\alpha$ ) in order to provide a theoretical basis for gene therapy of liver cancer. A recombinant eukaryotic expression vector (pcDNA3-MDA-7) containing human MDA-7 gene was constructed, which was then delivered to liver cancer cell line HepG2 and normal liver cell line L02. The positive cell clone was screened by G418. Reverse transcription quantitative polymerase chain reaction (RT-qPCR) was performed to confirm the occurrence of MDA-7 transcription in the transfected cells. The protein expression of MDA-7 was determined by western blot analysis. The effects of MDA-7 on liver cancer cell proliferation and apoptosis were investigated through MTT assay and flow cytometry by Annexin V-fluorescein isothiocyanate (FITC)/propidium iodide (PI) double-staining. The mitochondrial protein was extracted from the normal liver cell line L02 and liver cancer cell line HepG2 at 3 day post-culture, in which the alterations of anti-apoptotic B-cell lymphoma-2 (Bcl-2), pro-apoptotic $\mathrm{Bcl}-2$ associated $\mathrm{X}$ protein (Bax), mitochondria-released cytochrome $c$ and caspase 9 were determined by western blot analysis. pcDNA3-MDA-7 mediated the expression of foreign gene MDA-7 in HepG2 and L02 cells. MDA-7 promoted liver cancer cell apoptosis and inhibited cell proliferation; while no effect was exerted on normal liver cells, as determined by the MTT assay and flow cytometry. Relative to the L02 cells, the
\end{abstract}

Correspondence to: Dr Qingguo Mo, Department of Interventional Therapy, The Third Affiliated Hospital of Qiqihar Medical University, 27 Taishun Street, Tiefeng, Qiqihar, Heilongjiang 161000, P.R. China

E-mail: moqingguo1026@126.com

Key words: melanoma differentiation associated gene-7, recombinant eukaryotic expression vector, liver cancer, apoptosis, B-cell lymphoma-2 protein expression of Bcl-2 was downregulated in the HepG2 cells, while that of Bax, cytochrome $c$ and caspase 9 were upregulated. In the study, the eukaryotic expression vector pcDNA3-MDA-7 was successfully constructed, it can mediate the expression of MDA-7 in human liver cancer cells and normal liver cells and inhibits the proliferation of human liver cancer cells through the restored expression of mitochondrial pro-apoptotic Bcl-2.

\section{Introduction}

Liver cancer is acknowledged as a health burden worldwide, of which hepatocellular carcinoma (HCC) is the most frequently occuring type and the leading cause of death associated with cancer $(1,2)$. Additionally, it has been documented that liver cancer is one of the highest mortality tumors in China and its morbidity increases annually (3). As reported, the number of new cases augmented by 75\% from 1990 to 2015 . Besides, there are 854,000 newly diagnosed cases and 810,000 deaths reported in 2015, which makes the treatment of liver cancer still a priority in medical field across the world (2). According to a published report on cancer statistics in China, the upward trend concerning liver cancer may be attributed to the growth and aging of population (4). If patients with HCC cannot be diagnosed at an early stage, they may suffer from liver failure and cancer symptoms. Worse still, HCC at an advanced stage cannot be treated and patients commonly succumb to HCC after 3-6 months (5). Hereby, it is of significance to allocate resources for prevention, diagnosis, palliative care, screening and treatment to make sure proper interventions are effectively performed on patients with liver cancer (6).

Gene therapy is a promising treatment strategy for cancer by means of immunosuppressors, tumor suppressor genes, tumor- or tissue-specific promoter-driven suicide genes or antiangiogenic genes (7). However, gene therapy commonly induces apoptosis of normal cells along with the tumor cells, indicating that the tumor suppressive effects are not specific. Melanoma differentiation associated gene-7, also called interleukin 24 (IL-24) (MDA-7), is a tumor suppressor gene, which is firstly identified from melanoma cells (8). MDA-7 has been 
proved to exert selective tumor-suppressive effects without any normal cells affected (9). Besides, it has been demonstrated that MDA-7 can inhibit liver cancer cell proliferation (10), yet the mechanism has not been revealed. In addition, B-cell lymphoma 2 (Bcl-2), a recognized anti-apoptotic factor, can manipulate the intrinsic pathway in regulating the mitochondrial membrane permeability (11). Noteworthy, Bcl-2 has been pointed out to be effective in cancer therapies either alone or in combination with other therapies to facilitate the development of novel cancer therapies (12). Therefore, in the present study, we attempted to develop a liver cancer-specific gene therapy system by constructing recombinant eukaryotic expression vector pcDNA3-MDA-7 containing human MDA-7 to explore the underlying mechanism of the selective killing of liver cancer cells of MDA-7 with the involvement of Bcl-2 in an attempt to provide a theoretical basis for gene therapy of liver cancer.

The study was approved by the Ethics Committee of The Third Affiliated Hospital of Qiqihar Medical University (Qiqihar, China).

\section{Materials and methods}

Construction of eukaryotic expression vector (pcDNA3$M D A-7)$. The full-length polymerase chain reaction (PCR) product of MDA-7 (F: 5'-CTCTAGAGGGGCTGTGAAAGA CACTAT-3'; and R: 5'-CCTCGAGGGCATCCAGGTCAGA AGAA-3'; length: $630 \mathrm{bp}$ ) and the eukaryotic expression vector pcDNA3 were digested by the restriction endonucleases $\mathrm{XbaI}$ and XhoI (cat. nos. 1093A and 1094A), both from Takara Bio, Inc., (Otsu, Japan) followed by purification, recovery and ligation. The ligation product was transformed into DH5 $\alpha$ competent cells of Escherichia coli (preserved in our laboratory) by heat shock in water bath at $42^{\circ} \mathrm{C}$ for $90 \mathrm{sec}$. Next, culture in the plate containing ampicillin sodium (A7490; Solarbio Science \& Technology Co., Ltd., Beijing, China) for 12-16 h, single colony was selected for plasmid extraction and restriction enzyme digestion ( $\mathrm{Xba \textrm {I }}$ and $\mathrm{XhoI}$ ). The colony supplemented with clone of MDA-7 was screened out. The plasmid identified by restriction enzyme digestion was allowed to undergo sequencing and the correctly identified plasmid was used for subsequent experiments.

Cell line transfection using MDA-7. The pcDNA3-MDA-7 and empty vector pcDNA3 were transfected into HepG2 and L02 cell lines (cat. nos. HB8065 and CRL-12461; ATCC, Manassas, VA, USA) (both preserved in our laboratory) according to the instructions of Lipofectamine ${ }^{\mathrm{TM}} 3000$ (cat. no. L3000001; Thermo Fisher Scientific, Inc., Waltham, MA, USA), respectively. Then, the cells were cultured in medium containing 15\% calf serum (MB5175; Melone Pharmaceutical Co., Ltd., Dalian, China) at $37^{\circ} \mathrm{C}$ with $5 \% \mathrm{CO}_{2}$ for $48 \mathrm{~h}$. The medium was then replaced with selective culture medium containing G418 until the resistant clones were observed. The monoclone was selected for amplification culture. The transfected cell line with stable passage was established and stored at $-80^{\circ} \mathrm{C}$ for further use.

MDA-7 determination by reverse transcription quantitative PCR (RT-qPCR). The total RNA was extracted according to the instructions of the TRIzol RNA extraction kit (WLA088a, Wanlei Biotechnology Co., Ltd., Shenyang, China) for reverse transcription reaction. The reaction conditions are: $37^{\circ} \mathrm{C}$, for $15 \mathrm{~min} ; 98^{\circ} \mathrm{C}$ for $5 \mathrm{~min}$ and $4^{\circ} \mathrm{C}$ for $5 \mathrm{~min}$. The above reverse transcription product was used as a template for RT-qPCR cycle, and the $\beta$-actin was the internal reference. The RT-qPCR kit (RR037A, Takara Bio, Inc.) was used for reverse transcription and amplification. The required primers synthesized by Sangon Biotech were shown in Table I. The amplification conditions are $95^{\circ} \mathrm{C}$ for $5 \mathrm{sec}, 55^{\circ} \mathrm{C}$ for $10 \mathrm{sec}$ and $72^{\circ} \mathrm{C}$ for $15 \mathrm{sec}$. The RT-qPCR products were treated with $1 \%$ agarose gel electrophoresis for observation. All expression levels were calculated using the $2^{-\Delta \Delta \mathrm{Cq}}$ method (13).

MDA-7 determination by western blot analysis. The liver cancer cell lines of HepG2-pcDNA3-MDA-7, HepG2pcDNA3 and HepG2 along with the normal liver cell lines of L02-pcDNA3-MDA-7, L02-pcDNA3 and L02 were cultured until they reached the logarithmic growth phase. The culture medium was removed and the cells were washed with $1 \mathrm{ml}$ phosphate buffer saline (PBS) 3 times. The cells were lysed with $200 \mu \mathrm{l}$ protein lysis buffer (protease inhibitor was added at a ratio of 1:100) and incubated at $37^{\circ} \mathrm{C}$ for $2 \mathrm{~h}$. The cell-free supernatant was collected, followed by centrifugation $(12,000 \mathrm{x} \mathrm{g}$, at $4^{\circ} \mathrm{C}$ for $\left.5 \mathrm{~min}\right)$. The supernatant was the protein extract, which was quantified using the bicinchonininc acid (BCA) protein assay kit. The total protein in cells of each group was adjusted to $10 \mu \mathrm{g}$. A total of $15 \mathrm{ml}$ of sample was added. Following $10 \%$ sodium dodecyl sulphate-polyacrylamide gel electrophoresis (SDS-PAGE), the protein was transferred to the polyvinylidene fluoride (PVDF) membrane in a wet manner. The membrane was then blocked in 1X PBS containing 5\% skimmed milk for $4 \mathrm{~h}$ at room temperature and incubated with primary antibody, MDA-7 monoclonal antibody (cat. no. AF1965-SP; 1:1,000; R\&D Systems, Inc., Minneapolis, MN, USA) at $4^{\circ} \mathrm{C}$ overnight. After being washed with Tris-buffered saline with Tween-20 (TBST) 3 times ( 5 min per wash), the membrane was further incubated with the secondary antibody, rabbit anti-sheep IgG (cat. no. 6912-100; 1:2,000; BioVision, Inc., Milpitas, CA, USA) at room temperature for $2 \mathrm{~h}$, followed by washing with TBST for 3 times ( 5 min per wash). The membrane was developed using enhanced chemiluminescence (ECL, GE Healthcare Life Sciences, Logan, UT, USA).

Cell proliferation detected by 3-(4,5-dimethylthiazol-2yl)-2,5-diphenyltetrazolium bromide (MTT) assay. The liver cancer cell lines of HepG2-pcDNA3-MDA-7, HepG2pcDNA3 and HepG2 along with the normal liver cell lines of L02-pcDNA3-MDA-7, L02-pcDNA3 and L02 were cultured (3 wells per group) in serum-free medium until they reached the logarithmic growth phase. The period of growth in each group was controlled to be the same. The cells were then added into a 96-well plate at a density of $10^{4}$ cells/well (100 $\mu \mathrm{l} /$ well), cultured for $24 \mathrm{~h}$ and incubated with $20 \mu \mathrm{l} \mathrm{MTT}$ (5 mg/ml, 40201ES72, Shanghai Yeasen Biotechnology Co., Ltd., Shanghai, China) at $37^{\circ} \mathrm{C}$ for $4 \mathrm{~h}$. The culture medium was removed and $150 \mu \mathrm{l}$ dimethyl sulphoxide (DMSO) was added, followed by $15 \mathrm{~min}$ incubation at $37^{\circ} \mathrm{C}$. The absorbance value (A) was measured by a microplate reader (ELX-800; BioTek Instruments, Inc., Winooski, VT, USA) at 
Table I. Primer sequences for amplification of Mda7/IL-24.

\begin{tabular}{llc}
\hline Items & \multicolumn{1}{c}{ Forward primers } & \multicolumn{1}{c}{ Reverse primers } \\
\hline Mda7/IL-24 (90 bp) & 5'-GACTCGAGATGAATTTTCAACAGA-3' & 5'-GTCCCTCTGGTCCTGTAAG-3' \\
$\beta$-actin (219 bp) & 5'-CCTTCCTGGGCAATGGAGTCCT-3' & 5'-GGAACAATGATCTTGATCTT-3'
\end{tabular}

Mda7, melanoma differentiation associated gene-7; IL-24, interleukin 24.

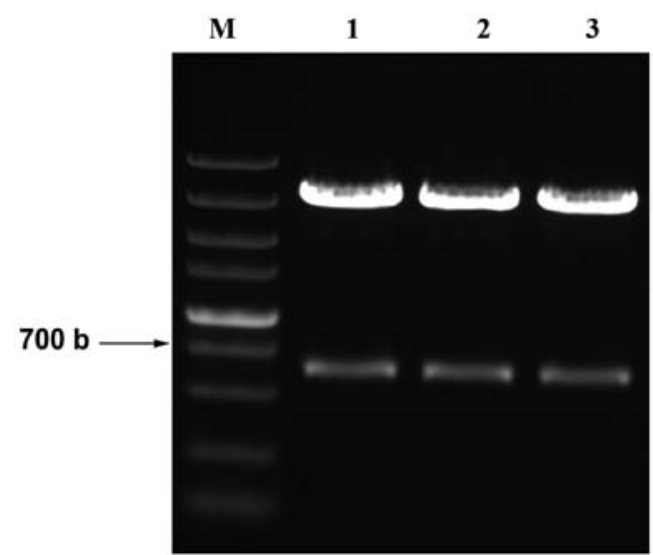

Figure 1. Successfully constructed pcDNA3-MDA-7. Lane 1, 2 and 3 are three randomly selected recombinant clones analyzed by restriction endonuclease digestion. M, DNA Marker; MDA-7, melanoma differentiation associated gene-7.

the wavelength of $540 \mathrm{~nm}$. The average value and the number of cells were calculated.

Cell apoptosis detected by flow cytometry. The liver cancer cell lines of HepG2-pcDNA3-MDA-7, HepG2-pcDNA3 and HepG2 along with the normal liver cell lines of L02-pcDNA3-MDA-7, L02-pcDNA3 and L02 were cultured in the 24-well plate and then treated with trypsin. The density of harvested cells was adjusted to $10^{6}$ cells $/ \mathrm{ml}$ and the binding buffer was added. Then, $100 \mu \mathrm{l}$ of the sample was added with $5 \mu \mathrm{l}$ Annexin V and $10 \mu \mathrm{l}$ propidium iodide (PI) dye (MA0220, Dalian Meilun Biotechnology Co., Ltd.). The sample was allowed to react for 15-20 min under conditions void of light and analyzed by flow cytometry (BD Accuri C6; BD Biosciences, San Jose, CA, USA). Cell Quest software (version 0.9.13 alpha; BD Biosciences) was used to acquire data, and FlowJo v10 software (FlowJo, LLC; Ashland, OR 97520, USA) was used to analyse.

Mitochondrial protein extraction. The liver cancer cell lines HepG2-pcDNA3-MDA-7, HepG2-pcDNA3 and HepG2 were cultured in the 24-well plate until they reached the logarithmic growth phase. The cells were collected and centrifuged $\left(600 \mathrm{x} \mathrm{g}\right.$ at $4^{\circ} \mathrm{C}$ for $8 \mathrm{~min}$ ) with the supernatant was removed. After re-suspension in $10 \mathrm{ml}$ pre-cooled PBS $\left(4^{\circ} \mathrm{C}\right)$, centrifugation was conducted again $\left(600 \mathrm{x} \mathrm{g}\right.$ at $4^{\circ} \mathrm{C}$ for $\left.8 \mathrm{~min}\right)$ and the supernatant was discarded. The cells were re-suspended in $1 \mathrm{ml}$ cytoplasm buffer. After standing on ice for $10 \mathrm{~min}$, the cell suspension was ground in a pre-cooled grinder for 10-20 min. Then, the well-ground fluid was transferred to a centrifuge tube for centrifugation $\left(600 \mathrm{x} \mathrm{g}\right.$ at $4^{\circ} \mathrm{C}$ for $\left.8 \mathrm{~min}\right)$ with the precipitate abandoned. The supernatant was subjected to centrifugation again $\left(10,000 \times \mathrm{g}\right.$ at $4^{\circ} \mathrm{C}$ for $\left.35 \mathrm{~min}\right)$. The obtained supernatant was considered as cytoplasmic protein. The obtained precipitate was dissolved in $100 \mu 1$ mitochondrion buffer and triturated with a pipette. The well-treated precipitate was considered as mitochondrial protein solution.

Mitochondrial protein determination by western blot analysis. The concentration of the obtained protein was determined by the protein analysis system (Bio-Rad Laboratories Inc., Hercules, CA, USA). The total protein in each group was adjusted to $10 \mu \mathrm{g}$. Following $10 \%$ SDS-PAGE, the protein was transferred onto the PVDF membrane in a wet manner. The membrane was then blocked in $1 \mathrm{X}$ PBS containing 5\% skimmed milk for $4 \mathrm{~h}$ at room temperature and incubated with the corresponding rabbit anti-human antibodies at $4^{\circ} \mathrm{C}$ overnight, including Bcl-2 (cat. no. LS-B6548; 1:1,000), Bcl-2 associated X protein (Bax, cat. no. LS-B1333; $1: 1,000$ ), caspase 9 (cat. no. LS-C148247; 1:1,000), cytochrome $c$ (cat. no. LS-C208738; 1:1,000) and actin (cat. no. LS-B11095; 1:1,000) all purchased from LifeSpan BioSciences, Inc. (Seattle WA, USA). After washing with TBST 3 times (5 min per wash), the membrane was further incubated with the secondary antibody, mouse anti-rabbit IgG (cat. no. LS-C60914; 1:3,000, GE Healthcare Life Sciences, Little Chalfont, UK) at room temperature for $2 \mathrm{~h}$, and then washed with TBST 3 times (5 min per wash) and developed by ECL (Amersham, Little Chalfont, Buckinghamshire, UK). All antibodies used in the procedure were purchased from LifeSpan BioSciences Inc., and diluted by the blocking fluid.

Statistical analysis. All data were calculated by SPSS Statistics 23.0 software (IBM Corp., Armonk, NY, USA). Test of significance was analyzed by independent sample t-test and analysis of variance. P-value $<0.05$ was considered to indicate a statistically significant difference.

\section{Results}

Successfully constructed pcDNA3-MDA-7 vector. After the construction of pcDNA3-MDA-7 vector, the extracted plasmid was digested by $\mathrm{XbaI}$ and $\mathrm{XhoI}$ and then confirmed by electrophoresis (Fig. 1). The correct bands (630 bp length) representing the fragment of MDA-7 of the three plasmids were identified. The three plasmids were then subjected to sequencing. Blast analysis was conducted to align the sequences and the match rate with the target sequence was $99.99 \%$. The results indicated that recombinant plasmid pcDNA3-MDA-7 was successfully constructed. 


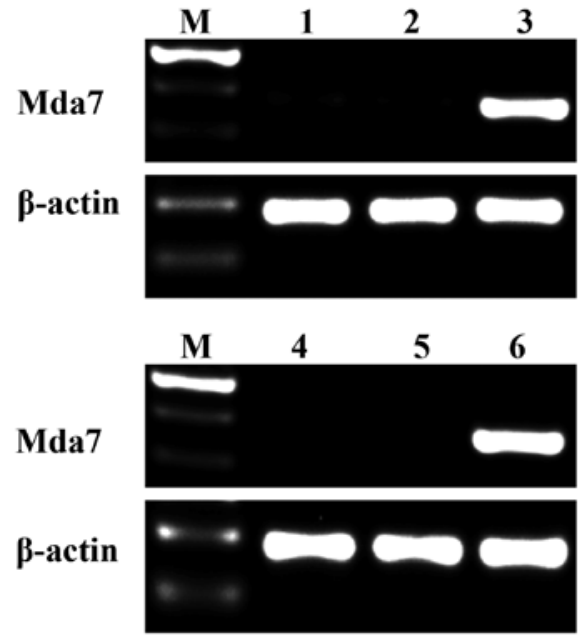

Figure 2. MDA-7 gene expressed in HepG2 and L02 cells transfected with pcDNA3-MDA-7. Lane 1, HepG2 cells; lane 2, HepG2-pcDNA3 cells; lane 3, HepG2-pcDNA3-MDA-7 cells; lane 4, L02 cells; lane 5, L02-pcDNA3 cells; lane 6, L02- pcDNA3-MDA-7 cells. M, DNA Marker; MDA-7, melanoma differentiation associated gene-7, also called interleukin 24 (IL-24).

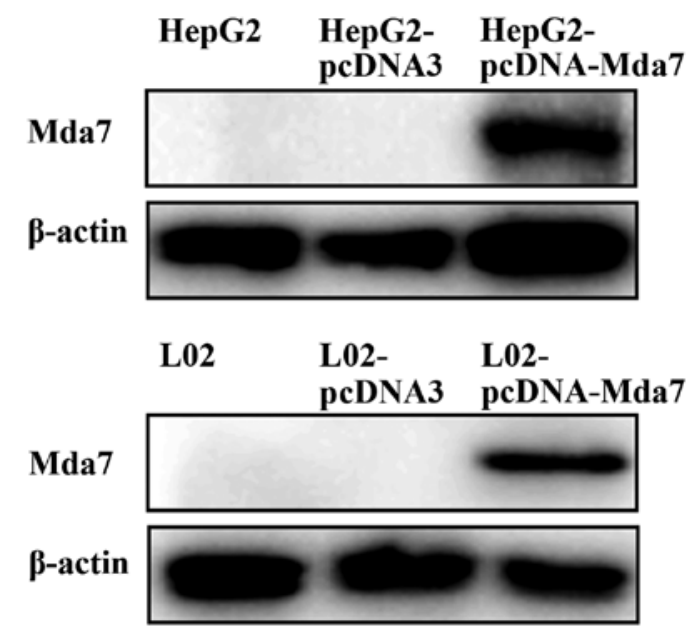

Figure 3. MDA-7 protein is expressed in $\mathrm{HepG} 2$ and $\mathrm{L} 02$ cells transfected with pcDNA3-MDA-7. MDA-7, melanoma differentiation associated gene-7, also called interleukin 24 (IL-24).

MDA-7 gene is expressed in HepG2 and LO2 cells transfected with $p c D N A 3-M D A-7$. The mRNA expression of MDA-7 in liver cells was identified by RT-qPCR (Fig. 2). The corresponding bands of MDA-7 were detected in HepG2 and L02 cells transfected with pcDNA3-MDA-7. However, no band was identified in cells transfected with pcDNA3 or the untransfected HepG2 and L02 cells. The findings indicated that MDA-7 gene could be expressed in HepG2 and L02 cells after transfection of pcDNA3-MDA-7.

MDA-7 protein is expressed in HepG2 and LO2 cells transfected with pcDNA3-MDA-7. The protein expression of MDA-7 in HepG2 and L02 cells was measured by western blot analysis (Fig. 3). The corresponding protein bands were detected in the HepG2 and L02 cells that were transfected with pcDNA3-MDA-7. While in the cells transfected with the pcDNA3 and the untransfected HepG2 and L02 cells, there
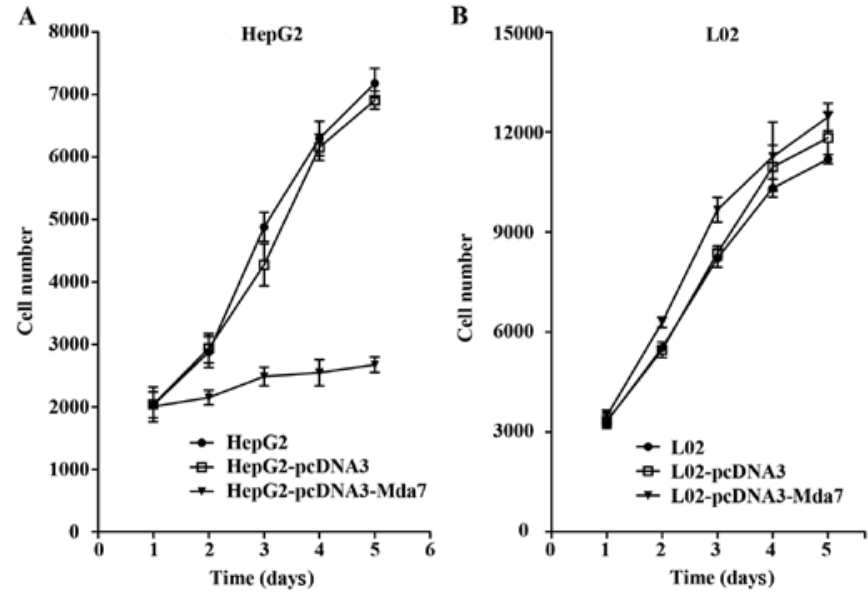

Figure 4. MDA-7 inhibits the proliferation of the liver cancer cells. (A) The number of the liver cancer cells (HepG2, HepG2-pcDNA3 and HepG2pcDNA3-MDA-7) following transfection of MDA-7. (B) The cell number of the normal liver cells (L02, L02-pcDNA3 and L02-pcDNA3-MDA-7) following transfection of MDA-7. MDA-7, melanoma differentiation associated gene-7, also called interleukin 24 (IL-24).

was no band in the corresponding region, indicating no MDA-7 protein expression. Therefore, it was concluded that MDA-7 protein can be expressed in HepG2 and L02 cells transfected with pcDNA3-MDA-7.

MDA-7 inhibits liver cancer cell proliferation. The effect of MDA-7 on the cell proliferation was evaluated through MTT assay (Fig. 4). The cell growth curves in each group were drawn. It was shown that pcDNA3-MDA-7 transfection could significantly inhibit the growth of the liver cancer cell line HepG2 $(\mathrm{P}<0.05)$ while no inhibitory effect was found in the normal liver cell line L02 ( $>>0.05)$. Thus, MDA-7 exerted inhibitory effect on the proliferation of liver cancer cells.

MDA-7 promotes liver cancer cell apoptosis. Cell apoptosis affected by MDA-7 was evaluated by flow cytometry. As depicted in Fig. 5, compared with the untransfected HepG2 and the pcDNA3 transfected HepG2 cells, the HepG2 cells transfected with pcDNA3-MDA-7 exhibited evidently higher apoptosis rate $(\mathrm{P}<0.05)$, which demonstrated that MDA-7 promoted HepG2 cell apoptosis. As for the normal liver cells, the apoptosis rates were all lower in L02 cells without transfection, with transfection of empty vector pcDNA3 or pcDNA3-MDA-7. Hence, the apoptosis of the liver cancer cells could be facilitated by MDA-7.

MDA-7 regulates the levels of mitochondrial apoptosis pathway-related proteins. The mechanism of MDA-7 on the suppression of liver cancer was subsequently investigated (Fig. 6). Compared with untransfected HepG2 cells and the empty vector-transfected HepG2 cells, the expression level of Bcl-2 (anti-apoptotic protein) decreased while that of Bax (pro-apoptotic protein), cytochrome $c$ and caspase 9 (marker proteins in the cell apoptosis signaling pathway) increased in HepG2 cells transfected with pcDNA3-MDA-7. Therefore, it was noted that MDA-7 suppresses the development of liver cancer by regulating the levels of the mitochondrial apoptosis pathway-related proteins. 

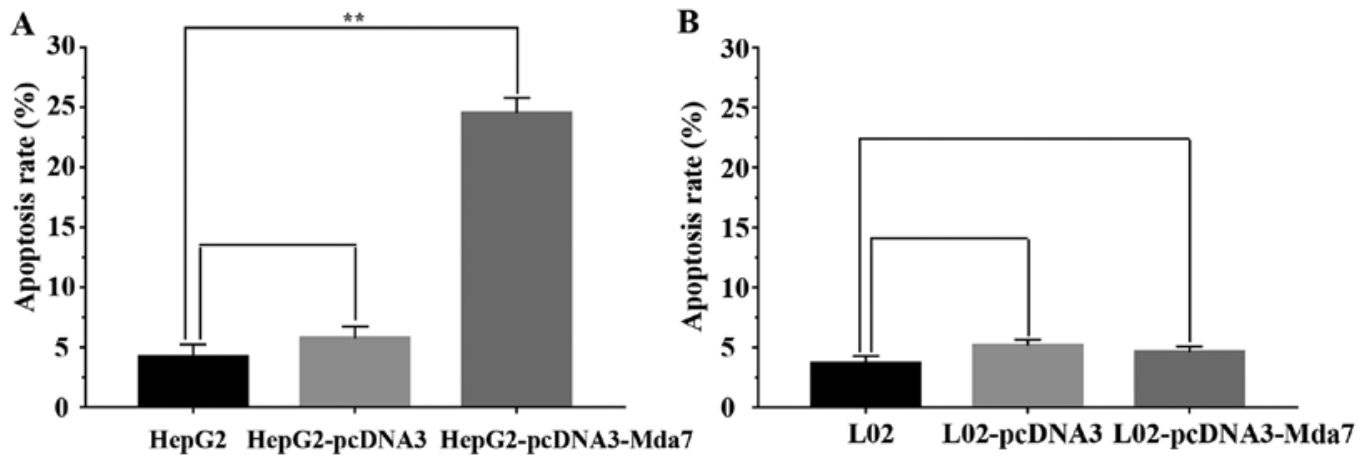

Figure 5. MDA-7 promotes apoptosis of the liver cancer cells. (A) The cell apoptosis rates of the liver cancer cells (HepG2, HepG2-pcDNA3 and HepG2pcDNA3-MDA-7) following transfection of MDA-7; compared with the untransfected HepG2 and the pcDNA3 transfected HepG2 cells, the HepG2 cells transfected with pcDNA3-MDA-7 exhibited evidently higher apoptosis rate $\left({ }^{* *} \mathrm{P}<0.05\right)$. (B) The cell apoptosis rates of the normal liver cells (L02, L02pcDNA3 and L02-pcDNA3-MDA-7) following transfection of MDA-7. MDA-7, melanoma differentiation associated gene-7, also called interleukin 24 (IL-24).

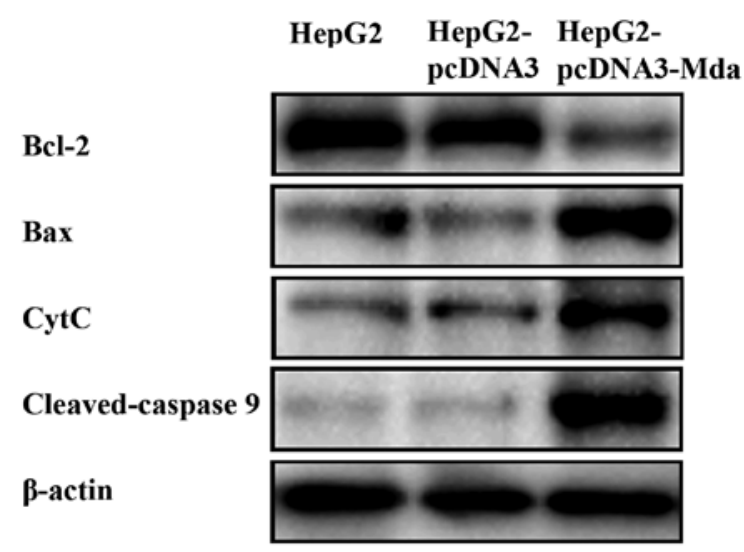

Figure 6. MDA-7 regulates the levels of mitochondrial apoptosis pathwayrelated proteins. MDA-7, melanoma differentiation associated gene-7, also called interleukin 24 (IL-24).

\section{Discussion}

Although the therapeutic level of liver cancer has exhibited continuous improvement in recent years, the overall survival rate of patients suffering from liver cancer still remains unfavorable. There is no doubt that the development of gene therapy and molecular oncology has brought great hope to cancer patients, including those with liver cancer. Multiple studies have provided evidence proving that MDA-7 can promote cell apoptosis in various types of tumor cells (14-17), highlighting its potential role as a targeting gene for tumor therapy. Therefore, the present study emphasized the effects of MDA-7 on liver cancer cells with a possible mechanism investigated, laying a theoretical foundation for MDA-7 as a candidate gene for the treatment of liver cancer.

Initially, the eukaryotic expression vector pcDNA3-MDA-7 was successfully constructed in the present study. The obtained results indicate that pcDNA3-MDA-7 could mediate the expression of MDA-7 in the liver cancer cell line HepG2 and the normal liver cell line L02. MDA-7 expression promoted apoptosis of liver cancer cell, but it had no obvious effect on the normal liver cells. Furthermore, the proliferation of the liver cancer cells was suppressed by upregulated MDA-7 while the normal liver cells were unaffected, indicating the specific functional role of MDA-7 on liver cancer cells and strongly supporting the therapeutic potential of MDA-7 for liver cancer. In addition, the changes of expression levels of the extracted mitochondrial proteins determined by western blot analysis suggested that $\mathrm{Bcl}-2$ expression was diminished significantly in HepG2 cells where Bax expression was obviously enhanced. Besides, apoptosis of the liver cancer cells was induced by stimulating the release of cytochrome $c$ from mitochondria and augmenting the expression of caspase 9 . All above-mentioned findings elucidated that MDA-7 could exert pro-apoptotic effects on HepG2 liver cancer cells through activation of the mitochondrial apoptotic pathway by downregulating Bcl-2 expression.

Wang et al have delivered Ad.VGFP/IL-24 to human liver cancer cell line SMMC-7721 and applied intratumoral injection in nude mice with liver cancer; as a result, the in vitro experiments revealed that Ad. VGFP/IL-24 could shorten the S phase of SMMC-7721 cells and block G2/M phase while the inhibitory effect on liver cancer cell growth was confirmed through in vivo experiments (18). Likewise, MDA-7 was found to promote liver cancer cell apoptosis according to results of MTT assay and flow cytometry in our study. It has been documented that MDA-7 can induce liver cancer cell apoptosis via the death receptor pathway and the endoplasmic reticulum stress signaling pathway (19). Consistently, our study indicated that MDA-7 induces apoptosis of the liver cancer cells via the mitochondrial apoptotic pathway in which cytochrome $c$ and caspase 9 were both upregulated, further leading to an in-depth understanding of tumor cell apoptosis induced by MDA-7. However, limitations are inevitable in the current study, for instance, the specific mechanism of liver cancer cell cycle entry that can be suppressed by MDA-7 is unclear and needs more exploration.

The anti-apoptotic protein Bcl-2, distributed on the cytoplasmic surface, endoplasmic reticulum and mitochondrial outer membrane of the nuclear membrane, has the ability to stabilize the mitochondrial membrane and block the release of caspase and cytochrome $c$ (20). On the contrary, Bax can promote the release of pro-apoptotic proteins including cytochrome $c$ by forming a mitochondrial transmembrane channel through combination with the mitochondrial membrane (21-23). The results of our study revealed that MDA-7 promoted apoptosis of liver cancer cells by decreasing the $\mathrm{Bcl}-2$ expression 
and increasing the expression of Bax and cytochrome $c$, which was in line with the aforementioned studies.

The current study leads to further understanding of the molecular mechanism of MDA-7-induced apoptosis in liver cancer cells, and provides a theoretical basis for the future clinical application of MDA-7 as a target therapy gene for liver cancer as well as other tumors.

\section{Acknowledgements}

Not applicable.

\section{Funding}

No funding was received.

\section{Availability of data and materials}

The datasets used and/or analyzed during the present study are available from the corresponding author on reasonable request.

\section{Authors' contributions}

QM and LL wrote the manuscript and performed PCR, western blot analysis and MTT assay. GB and TL were responsible for the construction of the eukaryotic expression vector. All authors read and approved the final manuscript.

\section{Ethics approval and consent to participate}

The study was approved by the Ethics Committee of The Third Affiliated Hospital of Qiqihar Medical University (Qiqihar, China).

\section{Patient consent for publication}

Not applicable.

\section{Competing interests}

The authors declare that they have no competing interests.

\section{References}

1. Chen Y, Liu R, Chu Z, Le B, Zeng H, Zhang X, Wu Q, Zhu G, Chen Y, Liu Y, et al: High glucose stimulates proliferative capacity of liver cancer cells possibly via O-GlcNAcylation-dependent transcriptional regulation of GJC1. J Cell Physiol 234: 606-618, 2018.

2. Rodríguez-Hernández MA, González R, de la Rosa AJ, Gallego P, Ordóñez R, Navarro-Villarán E, Contreras L, Rodríguez-Arribas M González-Gallego J, Álamo-Martínez JM, et al: Molecular characterization of autophagic and apoptotic signaling induced by sorafenib in liver cancer cells. J Cell Physiol 234: 692-708, 2018.

3. Wei KR, Yu X, Zheng RS, Peng XB, Zhang SW, Ji MF, Liang ZH, $\mathrm{Ou} \mathrm{ZX}$ and Chen WQ: Incidence and mortality of liver cancer in China, 2010. Chin J Cancer 33: 388-394, 2014.

4. Chen W, Zheng R, Baade PD, Zhang S, Zeng H, Bray F, Jemal A, Yu XQ and He J: Cancer statistics in China, 2015. CA Cancer J Clin 66: 115-132, 2016.

5. Bruix J, Reig M and Sherman M: Evidence-based diagnosis, staging, and treatment of patients with hepatocellular carcinoma. Gastroenterology 150: 835-853, 2016.

6. Global Burden of Disease Cancer Collaboration, Fitzmaurice C, Dicker D, Pain A, Hamavid H, Moradi-Lakeh M, MacIntyre MF, Allen C, Hansen G, Woodbrook R, Wolfe C, et al: The Global Burden of Cancer 2013. JAMA Oncol 1: 505-27, 2015.
7. Li W, Li DM, Chen K, Chen Z, Zong Y, Yin H, Xu ZK, Zhu Y, Gong FR and Tao M: Development of a gene therapy strategy to target hepatocellular carcinoma based inhibition of protein phosphatase $2 \mathrm{~A}$ using the $\alpha$-fetoprotein promoter enhancer and pgk promoter: An in vitro and in vivo study. BMC Cancer 12: 547,2012

8. Jiang H, Lin JJ, Su ZZ, Goldstein NI and Fisher PB: Subtraction hybridization identifies a novel melanoma differentiation associated gene, mda-7, modulated during human melanoma differentiation, growth and progression. Oncogene 11: 2477-2486, 1995.

9. Menezes ME, Bhoopathi P, Pradhan AK, Emdad L, Das SK, Guo C, Wang XY, Sarkar D and Fisher PB: Role of MDA-7/IL-24 a multifunction protein in human diseases. Adv Cancer Res 138: 143-182, 2018.

10. Wang CJ, Xue XB, Yi JL, Chen K, Zheng JW, Wang J, Zeng JP and $\mathrm{Xu}$ RH: Melanoma differentiation-associated gene-7, MDA-7/IL-24, selectively induces growth suppression, apoptosis in human hepatocellular carcinoma cell line HepG2 by replication-incompetent adenovirus vector. World J Gastroenterol 12: 1774-1779, 2006.

11. Hassan M, Watari H, AbuAlmaaty A, Ohba Y and Sakuragi N: Apoptosis and molecular targeting therapy in cancer. BioMed Res Int 2014: 150845, 2014.

12. Thomas S, Quinn BA, Das SK, Dash R, Emdad L, Dasgupta S, Wang XY, Dent P, Reed JC, Pellecchia M, et al: Targeting the Bcl-2 family for cancer therapy. Expert Opin Ther Targets 17: $61-75,2013$.

13. Livak KJ and Schmittgen TD: Analysis of relative gene expression data using real-time quantitative PCR and the 2(-Delta Delta C(T)) method. Methods 25: 402-408, 2001.

14. Bhoopathi P, Lee N, Pradhan AK, Shen XN, Das SK, Sarkar D, Emdad L and Fisher PB: mda-7/IL-24 induces cell death in neuroblastoma through a novel mechanism involving AIF and ATM. Cancer Res 76: 3572-3582, 2016.

15. Lin C, Liu H, Li L, Zhu Q, Liu H, Ji Z, Liao J, Lang J, Wu J and Fan J: MDA-7/IL-24 inhibits cell survival by inducing apoptosis in nasopharyngeal carcinoma. Int J Clin Exp Med 7: 4082-4090, 2014.

16. Menezes ME, Shen XN, Das SK, Emdad L, Guo C, Yuan F, Li YJ, Archer MC, Zacksenhaus E, Windle JJ, et al: MDA-7/IL-24 functions as a tumor suppressor gene in vivo in transgenic mouse models of breast cancer. Oncotarget 6: 36928-36942, 2015.

17. Park MA, Hamed HA, Mitchell C, Cruickshanks N, Dash R, Allegood J, Dmitriev IP, Tye G, Ogretmen B, Spiegel S, et al: A serotype 5/3 adenovirus expressing MDA-7/IL-24 infects renal carcinoma cells and promotes toxicity of agents that increase ROS and ceramide levels. Mol Pharmacol 79: 368-380, 2011.

18. Wang X, Ye Z, Zhong J, Xiang J and Yang J: Adenovirus-mediated IL-24 expression suppresses hepatocellular carcinoma growth via induction of cell apoptosis and cycling arrest and reduction of angiogenesis. Cancer Biother Radiopharm 22: 56-63, 2007.

19. Gupta P, Su ZZ, Lebedeva IV, Sarkar D, Sauane M, Emdad L, Bachelor MA, Grant S, Curiel DT and Dent P: mda-7/IL-24: Multifunctional cancer-specific apoptosis-inducing cytokine. Pharmacol Ther 111: 596-628, 2006.

20. Dai G, Zheng D, Guo W, Yang J and Cheng AY: Cinobufagin induces apoptosis in osteosarcoma cells via the mitochondria-mediated apoptotic pathway. Cell Physiol Biochem 46: 1134-1147, 2018

21. Ferrer PE, Frederick P, Gulbis JM, Dewson G and Kluck RM: Translocation of a Bak C-terminus mutant from cytosol to mitochondria to mediate cytochrome $\mathrm{C}$ release: Implications for Bak and Bax apoptotic function. PLoS One 7: e31510, 2012.

22. Mondal S, Bhattacharya K, Mallick A, Sangwan R and Mandal C: Bak compensated for Bax in p53-null cells to release cytochrome $\mathrm{c}$ for the initiation of mitochondrial signaling during Withanolide D-induced apoptosis. PLoS One 7: e34277, 2012.

23. Renault TT, Floros KV and Chipuk JE: BAK/BAX activation and cytochrome c release assays using isolated mitochondria. Methods 61: 146-155, 2013. International (CC BY-NC-ND 4.0) License. 